\title{
FSM BASED RE-TESTING METHODS
}

\author{
Khaled El-Fakih+, Nina Yevtushenko++ and Gregor v. Bochmann+++ \\ +Department of Computer Science, American University of Sharjah, UAE, kelfakih@aus.ac.ae \\ ++Tomsk State University, 36 Lenin Str., Tomsk, 634050, Russia, yevtushenko.rff@elefot.tsu.ru \\ +++ School of Information Technology and Engineering, University of Ottawa, Canada, \\ bochmann@site.uottawa.ca
}

\begin{abstract}
The selection of appropriate test cases is an important issue in software engineering. A number of methods are known for the selection of a test suite based on the specification and an implementation under test given in the form of a finite state machine (FSM). In realistic applications, this specification evolves incrementally throughout incorporating frequent modifications. In this paper, we adapt three well-known test derivation methods, namely the $\mathrm{W}, \mathrm{Wp}$, and HIS methods, for generating tests that would test the modified parts of the evolving specification. Application examples are provided.
\end{abstract}

Keywords: Conformance testing, finite state machines, test derivation, re-testing

\section{INTRODUCTION}

Many methods have been developed for deriving tests for a system represented by a Finite State Machine (FSM) model. The purpose of these tests is to determine whether an implementation of the system conforms to (i.e., is correct with respect to) its specification. Usually a conforming implementation is required to have the same $\mathrm{I} / \mathrm{O}$ behavior.

In realistic applications, maintaining a system modeled by a given specification machine involves modifying its specification as a result of changes in the users' requirements and designers implement incrementally these modifications. Testing the whole system implementation after each modification is considered expensive and time consuming. Therefore, it is important to generate tests (called re-tests) that would only test the modified

The original version of this chapter was revised: The copyright line was incorrect. This has been corrected. The Erratum to this chapter is available at DOI: 10.1007/978-0-387-35497-2_31 
parts of the implementation that correspond to the modified parts of its specification. This would reduce the maintenance cost of such a system, which is about two-thirds of the cost of the software production [10].

In this paper, we present test generation methods (called henceforth as retesting methods) that select tests (called re-tests) for testing the modified parts of the system specification, in order to check that these modifications were correctly implemented in the system implementation. Here we assume that the parts of the system implementation that correspond to the unmodified parts of the system specification are left intact. Moreover, we also reasonably assume that before modifying the system specification, its implementation was tested and found conforming to this specification. These methods are based on well-known test derivation methods called the W[1], Wp [3], and HIS [8] methods.

The problem of deriving re-testing sequences can be converted into the problem of test derivation from an FSM with a fault function [7] or from its generalization [5]. In this case, potential implementations are represented as all complete sub-machines of a given nondeterministic FSM that is called a mutation machine. However, the mutation machine from which re-testing sequences are derived, is special. Each unmodified specification transition is a deterministic transition of the mutation machine while each modified transition becomes chaotic; each pair (state, output) becomes possible as its tail state and output in a potential implementation. In other words, on one hand, the mutation machine has a number of deterministic transitions that can be used deriving a test suite, while on the other hand, a number of all possible paths that include modified transitions becomes exponential. Based on these features, we propose a proper technique for test derivation. First, we do not explicitly enumerate all possible implementation paths under an appropriate input sequence and secondly, we essentially use unmodified specification transitions that still remain deterministic in the mutation FSM. However, we always mention when our method delivers the same test suite as the methods for test derivation from an arbitrary mutation machine proposed in [7], [5].

This paper is organized as follows. Section 2, describes the finite state machine model, and Section 3 briefly describes the W, Wp, and HIS methods for generating tests from a given FSM specification. Based on these methods, our re-testing methods are presented in Section 4 with appropriate application examples. Section 5 concludes the paper. 


\section{FINITE STATE MACHINES}

A deterministic finite state machine is a 7-tuple $M=(S, X, Y, \delta$, $\lambda, D_{M}, s 1$ ), where: $S$ is a finite set of states, $s 1$ is the initial state, $X$ is a finite set of input symbols, $Y$ is a finite set of output symbols, $\delta$ is a next state (or transition) function: $\delta: D_{M}->S, \lambda$ is an output function: $\lambda: D_{M}->Y$, and $D_{M}$ is a specification domain: $D_{M} \subseteq S \times X$.

We use as in [3] the notation " $\left(s_{i}-x / y->s_{j}\right)$ " to indicate that the FSM $M$ at state $s_{i}$ responds with an output $y$ and makes the transition to the state $s_{j}$ when the input $x$ is applied. State $s_{i}$ is said to be the head or starting state of the transition, while $s_{j}$ is said to be the tail or ending state of the transition. If we are not interested in the output we write " $s i^{-v->s j}$ " when an input sequence $v$ is applied at state $s_{i}$. FSM $M$ is said to be completely specified or simply a complete FSM, if $D_{M}=S \times X$; otherwise, $M$ is said to be partially specified or simply a partial FSM. In the complete FSM, we omit the specification domain $D_{M}$, i.e. a complete FSM is a 6-tuple $M=(S, X, Y, \delta$, $\left.\lambda, s_{1}\right)$. The concatenation of sequences $v_{1}$ and $v_{2}$ is the sequence $v_{1} \cdot v_{2}$. For a given alphabet $Z, Z^{*}$ is used to denote the set of all finite words over $Z$. Let $V$ be a set of words over alphabet $Z$. The prefix closure of $V$, written $\operatorname{Pref}(V)$, consists of all the prefixes of each word in $V$, i.e. $\operatorname{Pref}(V)=\{\alpha \mid \exists \gamma(\alpha . \gamma \in$ $V)\}$. The set $V$ is prefix-closed if $\operatorname{Pref}(V)=V$.

Let $M_{S}=\left(S, X, Y, \delta_{S}, \lambda_{S}, D_{S}, s_{1}\right)$ and $M_{I}=\left(T, X, Y, \Delta_{I}, \Lambda_{I}, D_{I}, t_{1}\right)$ be two FSMs. In the following sections $M_{S}$ usually represents a protocol specification while $M_{I}$ denotes an implementation, and thus, FSM $M_{I}$ is further assumed to be complete. Given an input sequence $\alpha=x_{1} x_{2} \ldots x_{k} \in$ $X^{*}, \alpha$ is called a defined input sequence(DIS) at state $s_{i} \in S$, if there exist $k$ states $s_{i 1}, s_{i 2}, \ldots, s_{i k} \in S$ such that there is a sequence of specified transitions $s_{i-} x_{1->} s_{i 1}->\quad \ldots$ - $>s_{i}(k-1)-x_{k}>s_{i k}$ in the finite state machine $M_{S}$. Hereafter, DIS $\left(M S \mid s_{i}\right)$ will be used to denote the set of all the defined input sequences at state $s_{i}$ of machine $M S$.

We say that states $s_{i}$ of $M_{S}$ and $t_{j}$ of $M_{I}$ are compatible if $\operatorname{DIS}\left(M_{S} \mid s_{i}\right) \cap$ $\operatorname{DIS}\left(M_{I} \mid t_{j}\right)=\varnothing$ or if $\forall \alpha \in \mathbf{D I S}\left(M_{S} \mid s_{i}\right) \cap \mathbf{D I S}\left(M_{I} \mid t_{j}\right)$ it holds that $\lambda_{S}\left(s_{i}, \alpha\right)=$ $\Lambda_{I}(t j, \alpha)$. Otherwise; we say that states $s_{i}$ and $s_{j}$ are distinguishable. Input sequence $\alpha \in \mathbf{D I S}\left(M_{S} \mid s_{i}\right) \cap \mathbf{D I S}\left(M_{I} \mid t_{j}\right)$ such that $\lambda_{S}\left(s_{i}, \alpha\right) \neq \Lambda_{I}\left(t_{j}, \alpha\right)$ is said to distinguish the states $s_{i}$ and $t_{j}$. An FSM is said to be reduced if its states are pair-wise distinguishable. If the FSMs happen to be complete, then the definition of compatible states reduces to the definition of equivalent states (see for example, [4]). 


\section{REVIEW OF THE W, WP, AND HIS METHODS}

In the following section we briefly describe test derivation methods where the specification is given as a reduced FSM $M_{S}$ while an implementation under test (IUT) is modeled by a complete FSM $M_{I}$.

Let $t_{i}$ be a state of $M_{I}$ and $s_{j}$ be a state of $M_{S}$. Consider set $V$ of input sequences such that $V \subseteq \mathbf{D I S}\left(M_{S} \mid s_{j}\right)$. State $t_{i}$ is said to be equivalent to $s_{j}$ with respect to the set $V$ (written as $t_{i} \cong V s_{j}$ ), if $\Lambda_{I}\left(t_{i}, \alpha\right)=\lambda_{S}\left(s_{j}, \alpha\right)$ holds for any $\alpha \in V$. In other words, for each input sequence of $V$, a behavior of $M_{I}$ at state $t_{i}$ coincides with that of $M_{S}$ at state $s j$.

We say that $M_{I}$ conforms to $M_{S}$ if and only if $t_{1} \cong_{\operatorname{DIS}\left(M_{S} \mid s_{I}\right)} s_{1}$, where $t_{1}$ and $s 1$ are the initial states of $M_{I}$ and $M_{S}$, respectively. In other words, for each input sequence where a behavior of $M_{S}$ is defined, $M_{I}$ has the same behavior, i.e. the implementation is quasi-equivalent to the specification [4]. This conformance relation corresponds to the notion of weak conformance [9].

A set $Q$ of input sequences is called a state cover set of FSM $M_{S}$ if for each state $s_{i}$ of $S$, there is an input sequence $\alpha_{i} \in Q$ such that $s_{1}-\alpha_{i}->s_{i}$.

Usually the testing methods reviewed in this section use state identification facilities in order to check that each state and each transition defined in the specification also exists in the implementation. These facilities have certain input/output behaviors that can distinguish the states of an FSM. Given a reduced FSM $M_{S}$ and a state $s_{i} \in S$, a set $W_{i} \subseteq \mathbf{D I S}\left(M_{S} \mid s_{i}\right)$ of defined input sequences at state $s_{i}$ is called a state identifier of state $s_{i}$ if for any other state $s_{j}$ there exists $\alpha \in W_{i} \cap \operatorname{DIS}\left(M_{S} \mid s_{j}\right)$ such that $\lambda_{S}\left(s_{i}, \alpha\right) \neq$ $\lambda S(s j, \alpha)$. We now define a collection of state identifiers that has been named a family of harmonized identifiers [6], [8] or a separating family [11]. A separating family is a collection of state identifiers $W_{i}, s_{i} \in S$, which satisfy the condition that for any two states $s_{i}$, and $s_{j}, i \neq j$, there exist $\beta \in W_{i}$ and $\gamma$ $\in W_{j}$ which have common prefix $\alpha$ such that $\alpha \in \mathbf{D I S}\left(M_{S} \mid s_{i}\right) \cap \mathbf{D I S}\left(M_{S} \mid s_{j}\right)$, and $\lambda_{S}\left(s_{i}, \alpha\right) \neq \lambda S\left(s_{j}, \alpha\right)$. A separating family exists for any reduced (partial or complete) machine.

A characterization set of the FSM $M_{S}$, often simply called a $W$ set, is a set of input sequences which satisfies the following conditions:

(1) For any $s_{k} \in S, W \subseteq \mathbf{D I S}\left(M_{S} \mid s_{k}\right)$,

(2) For any two states $s_{i}$, and $s_{j}, i \neq j$, there exists $\beta \in W$ such that $\lambda_{S}\left(s_{i}, \beta\right) \neq \lambda_{S}\left(s_{j}, \beta\right)$.

A $W$ set always exists for a reduced completely specified machine. However, the $W$ set does not always exist for a reduced partially specified machine.

Given a specification reduced FSM $M_{S}=\left(S, X, Y, \delta_{S}, \lambda_{S}, D_{S}, s 1\right),|S|=\boldsymbol{n}$, and a complete implementation FSM $M_{I}=\left(T, X, Y, \Delta_{I}, \Lambda_{I}, t_{1}\right)$ such that 
$|T|=n$, let $W$ be a characterization set of $M_{S}$ (if exists) and $F=\left\{W_{1}, \ldots, W_{n}\right\}$ be a separating family of $M S$.

All the methods have two phases. In the first so-called state identification phase, they establish a one-to-one mapping $h_{S-I}: S \rightarrow T$ by the use of a characterization set $W$ or a separating family $F$. Given a prefix-closed state cover set $Q=\left\{\alpha_{1}, \alpha_{2}, \ldots, \alpha_{n}\right\}$ of the specification FSM, for each state $s_{j} \in S$, the state identification phase comprises the sequences: $r . \alpha_{j} . W_{j}$ (HIS method) or $r . \alpha_{j} . W$ (W and Wp methods).

We note that, if the specification FSM $M_{S}$ is partial, a characterization set $W$ may not exist; in this case, the $\mathrm{W}$ and $\mathrm{Wp}$ methods cannot be applied. However, the HIS method can be applied.

If FSM $M_{I}$ passes the state identification test sequences, then there exists one-to-one mapping $h_{S-I}: S \rightarrow T$ such that: $h\left(s_{j}\right)=t \Leftrightarrow s_{j} \cong w_{j} t$ in the HIS method, and $h\left(s_{j}\right)=t \Leftrightarrow s_{j} \cong_{W} t$ in the $\mathrm{W}$ and Wp methods.

The second so-called transition testing phase, assures that for each state $s \in S$, and input $x \in X$ that is defined at state $s$ the mapping $h S-I$ satisfies the following property:

$$
\lambda_{S}(s, x)=\Lambda_{I}\left(h_{S-I}(s), x\right) \text { and } h_{S-I}(\delta S(s, x))=\Delta_{I}\left(h_{S-I}(s), x\right)
$$

For this purpose, for each sequence $\alpha_{j} \in Q$ that takes the specification FSM to appropriate state $s_{j}$, and each $x \in X$ that takes the $M_{S}$ from state $s_{j}$ to state $s_{k}$, the testing transition phase includes the set of sequences:

$r . \alpha_{j} x . W_{k}$ in the HIS and Wp methods, where $W_{k}$ is a state identifier of the state $s_{k}$ in the specification FSM (for the Wp method, we have $W_{k} \subseteq W$ ) or

r. $\alpha_{j} . x . W$ in the $\mathrm{W}$ method

If FSM $M_{I}$ passes the test sequences of both testing phases, then it is quasi-equivalent to the specification FSM, i.e. is a conforming implementation. If the specification FSM is complete then the quasiequivalence relation reduces to the equivalence relation, i.e. the specification FSM and its conforming implementation have the same Input/Output behavior.

\section{FSM BASED RE-TESTING}

\subsection{Problem Definition}

Let the reduced FSM $M_{S}=\left(S, X, Y, \delta_{S}, \lambda_{S}, D_{S}, s_{1}\right)$ be the specification of a given system. We assume that the complete implementation FSM $M_{I}=\left(T, X, Y, \Delta_{I}, \Lambda_{I}, t_{1}\right)$ of $M_{S}$ with the same number of states has been tested and found conforming to $M S$. Therefore, there exists a one-to-one 
mapping $h_{S-I}: S \rightarrow T$ such that for each state $s \in S$ and input $x \in X$ that is defined at state $s,(\mathrm{X}-1)$ holds.

Let the reduced $M_{S}^{\prime}=\left(S, X, Y, \delta_{S}, \lambda_{S}, D_{S}^{\prime}, s_{1}\right)$ be the modified specification, and $M_{I}^{\prime}=\left(T, X, Y, \Delta_{I}, \Lambda_{I}, t_{1}\right)$ be the modified implementation that must conform to $M S^{\prime}$. We assume that only transitions corresponding to the modified parts of $M S^{\prime}$ have been changed in $M_{I}{ }^{\prime}$ and we want to generate test sequences for the modified parts of the system specification, in order to check that these modifications were implemented correctly in the modified implementation $M_{I}$. In other words, for each unmodified transition $\left(s_{j}-x / y->s_{k}\right)$ of the $M S^{\prime}$, we assume that transition $\left(h_{S-I}\left(s_{j}\right)-x / y->h_{S-I}\left(s_{k}\right)\right)$ has not been changed in the modified implementation $M_{I}$. We note that in case where new states are added (or deleted) to (or from) $M S$, we let $S^{\prime}$ denote the set of states of $M S^{\prime}$ and $T^{\prime}$ denote the set of states of $M^{\prime}$, respectively.

In general, we have the following types of modifications that can be made in $M_{S}$ and implemented by a designer in $M_{I}$ :

(1) outputs of some transitions are modified, (2) tail states of some transitions are modified, (3) outputs and tail states of some transitions are modified, (4) new transitions are added (5) some transitions are deleted, (6) new states are added, and (7) some states are deleted.

\subsection{The Re-testing Methods}

The re-testing methods adapted for the $\mathrm{W}, \mathrm{Wp}$, and HIS test derivation methods have also two phases. In the first phase, re-tests are selected in order to check (or re-identify) some states of the modified specification in the new implementation, and in the second re-testing phase, re-tests are selected to check each modified transition for correct output and tail state. Here, we examine different cases that can be used to generate short re-testing test sequences.

For convenience, hereafter, we use the input symbols $a$ and $b$ for unmodified transitions, and $x$ and $z$ for modified ones.

\subsection{Case-1: The Unmodified Part of the Modified Specification is Reduced}

Here we assume that the unmodified part $U P-M S^{\prime}$ of the modified specification $M S^{\prime}$ is reduced. Then, there exist state identifiers $W_{1}, \ldots, W_{n}$, which satisfy the following conditions: 1 ) each $W_{i}$ is a subset of the defined input sequences at state $s_{i} \in S$ in the $\left.U P-M S^{\prime}, 2\right)$ given two states $s_{i}$, and $s_{j}$, $i \neq j$, there exist sequences in $W_{i}$ and $W_{j}$ with the common prefix $\beta$ such that $\lambda s\left(s_{i}, \beta\right) \neq \lambda s(s j, \beta)$. 
We note that since the unmodified part of the specification can be partial, a characterization set $\mathrm{W}$ may not exist. However, we can always select state identifiers with the above conditions.

\subsubsection{General solution}

For each modified edge $\left(s^{-}-x / y->s_{k}\right)$, its corresponding re-testing test cases are formed as follows:

If we use the HIS or Wp methods: $r . \alpha_{j} . x . W_{k}$,

where $W_{k}$ is a state identifier of state $s k$.

If a characterization set $W$ exists and we use the $\mathrm{W}$ method: $r . \alpha_{j} . x . W$

Theorem 1. Given a modified specification $M S^{\prime}$ and its implementation $M_{I}{ }^{\prime}$, let the unmodified part of $M_{S}{ }^{\prime}$ be reduced and have state identifiers $W_{1}$, $\ldots, W_{n}$. If implementation $M_{I}{ }^{\prime}$ passes the re-testing test suite which consists of the union of the test cases over all modified transitions as given in Formulae (1-a) or (1-b), then the implementation $M_{I^{\prime}}$ is quasi-equivalent to $M S^{\prime}$.

We omit the proof of Theorem 1 since it is a particular case of Theorem 2.

We note that if the specification FSM is complete then the above case is a particular case of the advanced procedure in [7] since each state identifier is a so-called stable state identifier, i.e. is a state identifier of the corresponding state in each potential implementation.

\subsubsection{An Optimized Solution}

We observe that a sequence that distinguishes two states of the initial specification and traverses only unmodified transitions when applied at these states, also distinguishes the corresponding states of the modified implementation $M_{I}$ '. Moreover, when the unmodified part of the modified specification is reduced, the mapping $h_{S-I}(s): S \rightarrow T$ between the initial specification and its conforming implementation is the only candidate that can satisfy $(\mathrm{X}-1)$ for the modified specification and its implementation. Therefore, we do not need to re-identify states of the modified implementation. Moreover, the test suite constructed using the formulae of the general solution may be shortened if we use, when checking certain transitions, shorter state identifiers that pass through already tested transitions rather than those generated only from the unmodified part of the modified specification. In other words, instead of using state identifiers derived in advance, we can generate shorter state identifiers, as modified transitions are tested. For this purpose, we assume that a linear order "<" over modified transitions of the specification is given. This order satisfies 
the following property: If $\alpha_{r} . z \in Q$ is a prefix of $\alpha_{j} \in Q$, then for any two modified transitions $\left(s_{r}-z->s_{l}\right)$ and $\left(s_{j}-x->s_{k}\right)$, transition $\left(s_{r}-z->s_{l}\right)<\left(s_{j}-x->s_{k}\right)$. In this case, when checking a modified transition, we can use lower order transitions (or already checked transitions) to generate shorter re-testing sequences. The reason is that if the implementation at hand passes retesting sequences for transition $\left(s_{r}-z / y->s_{l}\right)$ then it has the corresponding transition $\left(h S-I\left(s_{r}\right)-z / y->h S-I(s l)\right)$, and this transition can be used for retesting a higher order transition. In this section, we illustrate by an example the advantage of using such a linear order. However, we do not discuss how to derive an order that provides the shortest re-testing sequences.

For each modified edge $\left(s_{j}-x->s_{k}\right)$, its corresponding re-testing test cases are formed as in Formulae (1-a) or (1-b). However, as a state identifier of state $s_{k}$, we use state identifier $W_{k}$ (or characterization set $W$ in W method) of the part of $M_{S}$ that comprises unmodified transitions or modified transitions $\left(s_{r}-z->s_{l}\right)$ where $\left(s_{r}-z->s_{l}\right)<\left(s_{j}-x->s_{k}\right)$. In other words, for testing transition $\left(s_{j}-x->s_{k}\right)$ the state identifier $W_{k}$ has sequences that if applied at state $s_{k}$ only traverse unmodified transitions or modified transitions $\left(s_{r} z->s_{l}\right)<\left(s_{j}-x->s_{k}\right)$. This allows, when checking a modified transition, the use of already re-checked transitions (or lower order transitions) in order to generate shorter state identifiers.

Theorem 2. Given a modified specification $M S^{\prime}$ and its implementation $M_{I}{ }^{\prime}$, let the unmodified part of $M_{S}{ }^{\prime}$ be reduced, and for each modified transition $\left(s_{j}-x-s_{k}\right) W_{k}$ is a state identifier of state $s_{k}$ in the part of $M_{S}$ that comprises unmodified transitions or modified transitions $\left(s_{r}-z->s_{l}\right)<\left(s_{j}-z-\right.$ $>s_{k}$ ). If implementation $M I^{\prime}$ passes the re-testing test suite which consists of the union of the test cases over all modified transitions as given in Formulae (1-a) or (1-b), then the implementation is quasi-equivalent to $M S^{\prime}$.

Proof. A proof of Theorem 2 is given in [2].

As an example for the general and optimized solution methods based on the HIS method, we consider the modified specification FSM $M_{1}$ shown in Fig. 1. FSM $M_{1}$ has the input set $X=\{a, b, c\}$, output set $Y=\left\{y_{1}, y_{2}, y_{3}\right\}$. The labels of the modified transitions are shown in bold. The unmodified part of FSM $M_{1}$ has a separating family $\left\{w_{1}, w_{2}, w_{3}, w_{4}\right\}$ of state identifiers, where $w_{1}=\{b b\}, w_{2}=\{b b\}, w_{3}=\{b b, c\}$ and $w_{4}=\{b b, c\}$. In fact, for each state $s_{i}$ of $M_{1}$ we have the following input/output sequences in response to $w_{i}$.

Table 1. Responses of $\mathrm{M}_{1}$ to state identifiers (if defined)

\begin{tabular}{|l|l|l|l|l|}
\hline & $s 1$ & $s 2$ & $s 3$ & $s 4$ \\
\hline$b b$ & $y_{1} y_{2}$ & $y_{2} y_{2}$ & $y_{2} y_{1}$ & $y_{2} y_{1}$ \\
\hline$c$ & & & $y_{2}$ & $y_{1}$ \\
\hline
\end{tabular}




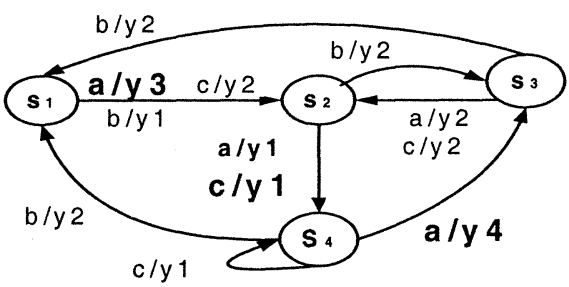

Figure 1. Specification $M_{1}$.

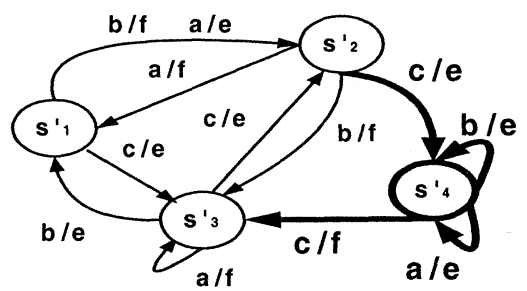

Figure 2. Specification $M S^{\prime}$.

None of the above state identifiers passes through a modified transition if applied at an appropriate state, and thus, the unmodified part of $M S^{\prime}$ is reduced. According to the general solution, in order to re-test the modified transitions, the following re-testing sequences of length 24 are generated using Formula (1-a): $\left\{r . \alpha_{1} \cdot a . w_{2}+\right.$ r. $\left.\alpha_{2} . c . w_{4}+r . \alpha_{4} . a . w_{3}\right\}=\{r . \varepsilon . a . b b, r . b . c . b b$, r.b.c.c, r.ba.a.bb, r.ba.a.c $\}$, where $\alpha_{1}=\varepsilon, \alpha_{2}=b$ and $\alpha_{4}=b a$ are the corresponding sequences of the state cover set .

We now use the following linear order over modified transitions $\left(s_{1}-a->s_{2}\right)<\left(s_{4} a->s_{3}\right)<\left(s_{2}-c->s_{4}\right)$. In order to re-test the modified transition $\left(s_{1}-a->s_{2}\right)$, the re-testing test sequence $\{r \cdot a \cdot w 2=r . a . b b\}$ is used. If the implementation at hand passes this sequence, then we have the following responses to the input $a$ :

At state $h_{S-I}\left(s_{1}\right): y_{3}$, at state $h_{S-I}\left(s_{2}\right): y_{1}$, and at state $h_{S-I}\left(s_{3}\right): y_{2}$,

Consequently, in order to re-test transition $\left(s 4-a-s_{s 3}\right)$, the re-testing test sequence $r . \alpha_{4} . a . a=r . b . a . a . a$ will be enough instead of $r . \alpha_{4} . a_{.} w_{3}$, since $r . \alpha_{4}$ reaches state $s 4$ through unmodified transitions and if afterwards, the modified implementation produces the expected output $y_{4}$ to the input symbol $a$, then $a$ becomes a distinguishing sequence for the part of the specification comprising unmodified transitions and transitions $\left(s_{1}-a->s_{2}\right)$ and (s4-a->s3), where for the last transition only its output has been checked; therefore, $a$ is a distinguishing sequence for the corresponding part of the modified implementation.

Afterwards, in order to check the ending state $s 4$ of the modified transition $(s 2-c->s 4)$, the distinguishing sequence $a$ can be used instead of the previous state identifier $w 4=\{b b, c\}$. Hence, the re-testing test sequence of this transition is r. $\alpha_{2} . c . a=r . b . c . a$. Therefore, according to the optimized solution method, in order to re-test the modified transitions, the following retesting sequences are generated using the above linear order and Formulae $(1-a):\{r . a . b b+$ r.b.a.a.a +r.b.c.a $\}$.

The total length of these sequences is 13 , where the total length of those generated using Formula (1-a) of the general solution method is 24. The HIS method generates a test suite of length 51 if the whole specification of $M_{S}{ }^{\prime}$ is considered for test derivation. 


\subsection{Case-2: Each State of the Modified Specification is Reachable Through Unmodified Transitions and the Unmodified Part is Not Reduced.}

In some cases, the unmodified part of the modified specification $M S^{\prime}$ is not reduced. However, each state of $M S^{\prime}$ is reachable through some unmodified transitions. Since each state of $M S^{\prime}$ can be reached through unmodified transitions, the only possible correct mapping between the states of $M S^{\prime}$ and $M I^{\prime}$ is the old mapping established between the states of $M S$ and $M_{I}$. Therefore, in order to check that this mapping still holds for the states of the modified specification and implementation the only states that have state identifiers passing through modified transitions have to be re-identified in the new implementation. Moreover, in order to re-identify such a state, it is enough to apply only the sequences of the corresponding state identifier that pass through modified transitions.

Let $Q$ be a prefix-closed state cover set such that its sequences do not traverse modified transitions if applied at the initial state of $M S^{\prime}$. Let also $F=$ $\left\{W_{1}, \ldots, W_{n}\right\}$ be a separating family of the modified specification, and $W$ be a characterization set (if exists).

i) State re-identification phase

For each state $s_{r}$ such that some sequences of $W_{r}$ traverse modified transitions if applied at $s_{r}$ the state re-identification sequences are formed as follows:

$$
r . \alpha_{r} \cdot W_{r}
$$

where $W_{r} \subseteq W_{r}$ (or $W_{r}^{\prime} \subseteq W$ for the $\mathrm{W}$ and $\mathrm{Wp}$ methods) comprises each sequence of the state identifier $W_{r}$ (of the characterization set $W$ ) that, if applied at state $s_{r}$ of the modified specification, traverses a modified transition. We note that each state of $M S^{\prime}$ for which all sequences of the state identifier traverse only unmodified transitions, does not need to be reidentified.

\section{ii) Re-testing modified transitions phase}

For each modified edge $\left(s_{j}-x->s_{k}\right)$, its corresponding re-testing test sequences are formed as shown in Formulae (1-a) and (1-b).

Theorem 3. Given the modified specification $M S^{\prime}$ and its implementation $M_{I}^{\prime}$, let $F=\left\{W_{1}, \ldots, W_{n}\right\}$ be a separating family of $M_{S^{\prime}}$ and $W$ be a characterization set of $M_{S}^{\prime}$ (if exists). Let also $Q$ be a prefix-closed state cover set of $M_{S^{\prime}}$ such that each sequence of the set $Q$ does not traverse a modified transition if applied at the initial state. If implementation $M_{I^{\prime}}$ passes the re-testing test suite which is the union of the re-testing test sequences given in Formula (2-1a) and Formulae (1-a) or (1-b), then the implementation is quasi-equivalent to $M S^{\prime}$.

We omit the proof of Theorem 3 since it is a particular case of Theorem 4. 
We note that union of the test cases given in Formula (2-1a) and Formula (1-a) coincides with the test suite returned by procedures in [7], [5], since both methods return a test suite without input sequences that traverse only unmodified transitions. However, here we underline the advantage of selecting a state cover set and state identifiers with sequences that do not traverse any modified transition. In this case, the old image of a corresponding state of a modified specification must be preserved and therefore, there is no need to check unmodified transitions at the corresponding state.

\subsection{Case-3: Some States are only Reachable Through Modified Transitions}

In some cases, the unmodified part of the modified specification $M S^{\prime}$ is not reduced and some states of $M S^{\prime}$ are only reachable through modified transitions. This case always holds when additional states are introduced when modifying the specification. Here, for the subset of states, say $S_{r-m}$ of the modified specification that are only reachable through modified transitions, the old conforming mapping might not be preserved between the new specification and its implementation, i.e. some $s_{k} \in S_{r-m}$ of the modified specification might be mapped to a new state of its implementation (say $t_{l} \in T_{r-m}$ ), different from $t_{k}$. Each such state must be re-identified in the new implementation and moreover, differently from former two cases, we have to check unmodified transitions from this state.

As an example, we modify the specification $M_{S}$ shown in the upper part of Fig. 3 and obtain the FSM $M S^{\prime}$ shown in the upper of Fig. 4. The modified transitions are shown as bold lines. We note that the mapping between states of $M_{S}$ and its conforming implementation $M_{I}$, shown in the lower part of Fig. 3, is $h_{S-I}\left(s_{k}\right)=t_{k}$ for $k=1, \ldots, 4$. Moreover, we let $M_{I}$, shown in the lower part of Fig. 4, be the implementation of $M S^{\prime} . M S^{\prime}$ has $W=\{a a\}$ as a characterization set which is a state identifier of each state. In fact, we have the following output responses to $a a$. For state $s_{1}, x x$. For state $s_{2}, x y$. For state $s 3, y y$, and for state $s 4, y x$. 


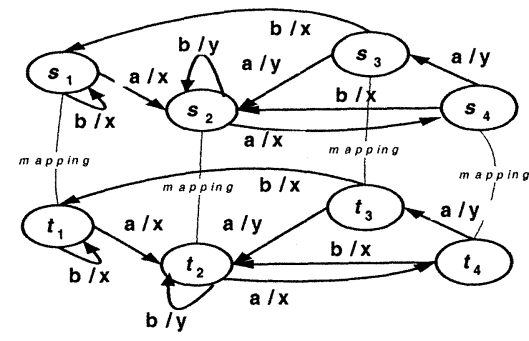

Figure 3. $M_{S}$ and $M_{I}$

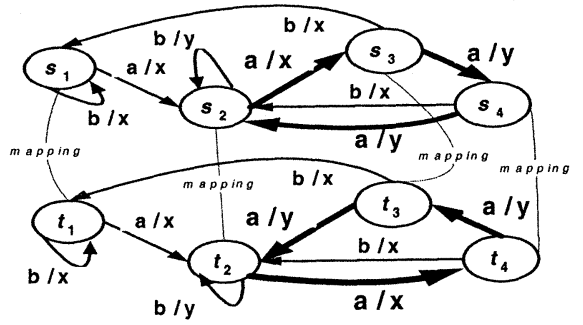

Figure 4. $M_{S}{ }^{\prime}$ and $M_{I}$ '.

We note that the modified implementation state $t_{4}$ of $M I^{\prime}$ has the output response $y y$ to $a a$, i.e. state $s 3$ of $M S^{\prime}$ is $W$-equivalent to $t 4$ of $M I^{\prime}$ while state $s 4$ of $M I^{\prime}$ is $W$-equivalent to $t_{3}$ of $M I^{\prime}$. States $t_{1}$ and $t_{2}$ of $M I^{\prime}$ are $W$ equivalent to $s_{1}$ and $s_{2}$ of $M_{I}{ }^{\prime}$. The mapping is preserved for the modified transitions from states $t_{3}$ and $t 4$ under input $a$. However, it is not preserved for the unmodified transitions at states $s 3$ of $M S^{\prime}$ and $s 4$ of $M S^{\prime}$ under input b, i.e. $M_{I^{\prime}}$ is a wrong implementation of $M S^{\prime}$. Therefore, appropriate unmodified transitions also need to be checked in order to kill such a wrong mapping.

As in the previous section, we select a prefix-closed state cover set with the following property. Given state $s j \in S$ of $M S^{\prime}$ reachable through unmodified transitions, we select the sequence $\alpha_{j} \in Q$ that does not traverse any modified transition.

The re-testing method has two phases. In the first phase, some states of the modified specification are re-identified in the new implementation. It may occur that for some states of the modified specification that are only reachable through modified transitions their old image must still be preserved in the new implementation. In particular, those are the states that have a so-called stable identifier [7] that distinguishes a state from any other state in each possible implementation. For each such state, we derive a state identifier (if it exists) that kills, through the re-identification phase, implementations where the state has a new image. We start from the set of states that are reachable through unmodified transitions. As in Case-2, the old mapping must still be valid for these states and only modified transitions from these states need to be checked. Moreover, given such a state, if the sequences of its state identifier do not traverse modified transitions then the state does not need to be re-identified. Otherwise, we select state reidentification sequences using Formula (2-1a) of Case-2, where in order to check the new mapping of a state, say $s j$, we concatenate the sequence $r . \alpha_{j}$ with each sequence of the state identifier of the $s j$ that passes through a modified transition. Then, we iteratively identify all other states for which the old mapping must be preserved; however, their state identifiers are 
derived in a proper way as described below. Afterwards, since each remaining state, say $s_{j} \in S_{r-m}$, of the modified specification could have a new image, i.e. $s_{j}$ could be mapped to say $t_{k} \in T_{r-m}$ instead of the old image $h_{S-I}\left(s_{j}\right)=t_{j}$, re-tests are selected to re-identify the image of $s_{j}$ in the new implementation, i.e. check (or establish) that $s_{j}$ is W-equivalent to $t_{k}$, and to check that this mapping is conforming. In order to re-identify $s j$, re-tests are selected by concatenating $r . \alpha_{j}$ with each sequence in the state identifier of state $s_{j}$ including sequences which traverse only unmodified transitions Moreover, in order to check that this mapping is a valid one (i.e. kill wrong mappings), re-tests are selected to check each outgoing transition from $s j$ for correct output and ending state in the new implementation.

In order to implement the above steps, we determine a subset $S_{u}$ of the set of states of the modified specification such that for the states in $S_{u}$ the old mapping between the states of the modified specification and its conforming modified implementation must still be preserved. The set $S_{u}$ enjoys a nice property. For each state in $S_{u}$, we do not need to check its outgoing unmodified transitions. In the following paragraph, we determine which states may be in the set $S_{u}$ and derive the set $S_{u}$ together with a separating family $F=\left\{W_{1}, \ldots, W_{n}\right\}$ (or characterization set $W$ ) so that if the implementation passes the re-identification test sequences, then there exists one-to-one mapping $h: S \rightarrow T$ such that the following property holds.

For each state $s_{i} \in S_{u}$ we have: $s_{i} \cong w_{i} t \Leftrightarrow t=h_{S-I}\left(s_{i}\right)$.

First, we add to the empty set $S_{u}$ each state $s j$ that is reachable from the initial state through unmodified transitions. As in Case-2, the images of these states have to be still preserved in the new implementation. Then, for state $s_{j}$ and each state $s_{i} \in S, s_{i} \neq s_{j}$, we include in the state identifiers $W_{j}$ and $W_{i}$ a sequence that distinguishes the states $s_{j}$ and $s_{i}$ in the modified specification. We note that, as discussed for Case-2, we recommend, while building the state identifier $W_{j}$, to select the sequences that do not pass through modified transitions if applied at state $s j$, since we do not need to apply these sequences while re-identifying $s j$.

Afterwards, we iteratively include in $S_{u}$ each state $s_{j} \in S S_{u}$, such that for each state $s_{i} \in S S_{u}, s_{i} \neq s_{j}$, there exists sequence $\beta_{i j}$ that does not traverse modified transitions if applied at states $s_{i}$ and $s_{j}$ and $\lambda_{S}\left(s_{i}, \beta_{i j}\right) \neq \lambda_{S}\left(s_{j}, \beta_{i j}\right)$, or there exists input $x$ such that transitions $\left(s_{j}-x->s_{k}\right)$ and $\left(s_{i}-x->s_{r}\right)$ are unmodified, $s_{k} \neq s_{r}$ and $s_{k}, s_{r} \in S_{u}$. In the former case, we include sequence $\beta_{i j}$ in $W_{i}$ and $W_{j}$. Since $\beta_{i j}$ does not traverse modified transitions if applied at states $s_{i}$ and $s_{j}$ we have that $\Lambda_{I}\left(h_{S-I}\left(s_{i}\right), \beta_{i j}\right)=\lambda_{S}\left(s_{i}, \beta_{i j}\right)$, and $\lambda_{S}\left(s_{j}, \beta_{i j}\right) \neq$ $\Lambda_{I}\left(h_{S-I}\left(s_{i}\right), \beta_{i j}\right)$. Thus, if $\beta_{i j}$ is included into $W_{i}$ and $W_{j}$ and the implementation passes the corresponding state re-identification sequences, then $s_{j}$ is not $W$-equivalent to $h_{S-I}\left(s_{i}\right)$ (i.e $h_{S-I}\left(s_{j}\right) \neq h_{S-I}\left(s_{i}\right)$ ). In the latter case, we include into $W_{i}$ and $W_{j}$ the sequence $x \beta$ where $\beta$ is a common 
prefix of the appropriate sequences in $W_{i}$ and $W_{j}$ such that $\lambda_{S}\left(s_{k}, \beta\right) \neq$ $\lambda_{S}\left(s_{r}, \beta\right)$. Thus if $\Lambda_{I}\left(h_{S-I}\left(s_{i}\right), x . \beta\right)=\lambda_{S}\left(s_{i}, x . \beta\right)$, then $\lambda_{S}\left(s_{j}, x . \beta\right) \neq \Lambda_{I}\left(h_{S-I}\left(s_{i}\right)\right.$, $x . \beta$ ). If $x . \beta$ is included in $W_{i}$ and $W_{j}$ and the implementation passes the corresponding state re-identification sequences, then $s_{j}$ is not $W$-equivalent to $h_{S-I}\left(s_{i}\right)$. Due to the definition of state identifiers for states in $S_{u}$, such a sequence exists. If any sequence of each state identifier is defined at each state then we derive the set $W$ as the union of all state identifiers. We note that in order to kill for $s_{j}$ any mapping $h_{I}$ where $h_{I}\left(s_{j}\right) \neq h_{S-I}\left(s_{j}\right)$, the corresponding state re-identification sequences are derived by concatenating $r . \alpha_{j}$ with every sequence of the set $W_{j}$ (or $W$ for the $\mathrm{Wp}$ and $W$ methods).

Finally, we derive state identifiers for the remaining states in $S \backslash S_{u}$. For each state $s_{j}$ in $S S_{u}$ and for each state $s_{i} \in S, s_{i} \neq s_{j}$, we include a sequence $\beta_{i j}$ in $W_{i}$ and $W_{j}$ (if it does not already exist) such that $\lambda_{s}\left(s_{i}, \beta_{i j}\right) \neq \lambda_{s}\left(s_{j}\right.$, $\beta_{i j}$ ). In order to re-identify $s j$ in the new implementation and kill its possible wrong images, the corresponding re-testing sequences include all reidentification sequences and re-testing sequences for testing all outgoing transitions from state $s j$.

The characterization set $W$ (for the $\mathrm{W}$, and $\mathrm{Wp}$ methods) can be obtained as the union of state identifiers $W_{i}, i=1, \ldots, n$ (if possible). We note that in order to reduce the number of transitions which need to be checked we use another technique than that based on stable state identifiers [7]. The main idea behind our approach is based on the observation that for each state reachable through unmodified transitions and some other states, the old image must be preserved in each conforming modified implementation. Our technique can also be used to reduce a test suite derived from a mutation machine [5] if the latter has many deterministic transitions.

\section{i) Phase of state re-identification}

For each state $s j$ of the modified specification that needs to be reidentified in the new implementation, we derive its state re-identification test sequences as follows:

If $\alpha_{j}$ does not traverse a modified transition, the re-identification sequences are formed as in Formula (2-1a).

If $\alpha_{j}$ traverses a modified transition then there are test sequences
$r . \alpha_{j} . W_{j}$ (HIS method);
r. $\alpha_{j} . W$ (W and Wp methods).

Every sequence of the set $W_{j}$ (or $W$ ) must be applied after $\alpha_{j}$, whether the sequence applied at state $s_{j}$ traverses a modified transition or not.

\section{ii) Phase of re-testing modified transitions}

For each modified edge $\left(s_{j}-x-s_{k}\right)$, where $s_{j} \in S_{u}$, its corresponding test cases are formed as in Formulae (1-a) or (1-b). For each state $s_{j} \notin S_{u}$, 
Formula (1-a) or (1-b) are applied for each outgoing transition from state $s_{j}$ including those which are unmodified.

Theorem 4. Given the modified specification $M S^{\prime}$ and implementation $M_{I}$, let $Q$ be a prefix-closed state cover set of $M_{S}{ }^{\prime}$ and $F=\left\{W_{1}, \ldots, W_{n}\right\}$ and $W$ be a separating family and a characterization set (if exists) of the modified specification $M S^{\prime}$ derived as described above. If implementation $M I^{\prime}$ passes the re-testing test suite derived for Case-3, then the implementation is quasi-equivalent to $M S^{\prime}$.

Proof. As we demonstrated by the example, in Case 3, a one-to-one mapping $h: S \rightarrow T$ such that state $s_{i}$ of $M S^{\prime}$ is $W_{i}$-equivalent to state $h\left(s_{i}\right)$ of $M_{I}{ }^{\prime}$ can be different from $h_{S-I}$. We first need to check whether the one-toone mapping $h$ exists at all.

We consider a relation $h \in S \times T$ such that: $\left(s_{j}, t_{j}\right) \in h \Leftrightarrow s_{j} \cong w_{j} t_{j}$. If the implementation passes the re-identification test cases given by Formulae (2-1a), (3-1a) and (3-1b) then $h$ is a one-to-one mapping $h: S \rightarrow T$. We next show that $h\left(s_{j}\right)=h_{S-I}\left(s_{j}\right)$ holds for each $s_{j} \in S_{u}$.

Given state $s_{j} \in S$ of $M S^{\prime}$ such that the sequence $\alpha_{j} \in Q$ does not traverse modified transitions, if $M_{I}{ }^{\prime}$ passes the test sequences given in Formulae (31a) then the state $h S-I\left(s_{j}\right)=h\left(s_{j}\right)$. The initial state $s_{1}$ is in the set $S_{u}$, i.e. the base of induction holds.

Let us assume that $h(s)=h S-I(s)$ holds for each state $s$ of a current set $S_{u}$ and that state $s_{j}$ is the next state we are going to include into $S_{u}$ using the procedure described above. Since $h$ is a one-to-one mapping, for each state $s \in S_{u}$ it holds that $h\left(s_{j}\right) \neq h S$-I $(s)$. On the other hand, for each state $s_{i} \in S \backslash S_{U}$, $i \neq j$, by construction of the state identifier, $\exists$ a sequence $\beta_{i j} \in W_{j} \cap W_{i}$ such that $\beta_{i j}$ does not traverse modified transitions if applied at states $s_{i}$ or $s_{j}$ and $\lambda_{S}\left(s_{i}, \beta_{i j}\right) \neq \lambda_{S}\left(s_{j}, \beta_{i j}\right)$, or $\exists$ a sequence $x \beta \in W_{j} \cap W_{i}$ such that the final states of unmodified transitions $\left(s-x->s_{k}\right)$ and $\left(s_{i}-x->s_{r}\right)$ are different and $\lambda_{S}\left(s_{k}, \beta\right) \neq \lambda_{S}\left(s_{r}, \beta\right)$. In the former case, $M_{I}{ }^{\prime}$ has different output responses to the sequence $\beta_{i j} \in W_{j} \cap W_{i}$ at the states $h\left(s_{j}\right)$ and $h_{S-I}\left(s_{i}\right)$, i.e. $h\left(s_{j}\right) \neq h_{S-I}\left(s_{i}\right)$. In the latter case, $M_{I}{ }^{\prime}$ at states $h S-I\left(s_{k}\right)$ and $h_{S-I}\left(s_{r}\right)$ has different output responses to the sequence $\beta \in W_{k} \cap W_{r}$, i.e. $M_{I}{ }^{\prime}$ has different output responses to the sequence $x \beta \in W_{k} \cap W_{r}$ at the states $h\left(s_{j}\right)$ and $h S-I\left(s_{i}\right)$, i.e. $h\left(s_{j}\right) \neq h_{S-I}\left(s_{i}\right)$. Therefore, by induction, $h\left(s_{j}\right)=h S_{-I}\left(s_{j}\right)$ for each state $s_{j} \in S_{u}$.

For each unmodified transition $\left(s_{j}-a->s_{l}\right)$ from state $s_{j} \in S_{u}$ it holds that $\lambda_{S}\left(s_{j}, a\right)=\Lambda_{I}\left(h_{S-I}\left(s_{j}\right)\right)$ and $h_{S-I}\left(s_{l}\right)=\Delta\left(h_{S-I}\left(s_{j}\right), a\right)$.

If $M I^{\prime}$ passes the test cases $r . \alpha_{j} . x . W_{k}$ for a modified transition $\left(s_{j}-x->s_{k}\right)$ then $\lambda_{S}\left(s_{j}, x\right)=\Lambda_{I}\left(h_{S-I}\left(s_{j}\right), x\right)$ and the ending state of the transition $\left(h_{S-I} I\left(s_{j}\right)-x\right.$ $\rightarrow t_{k}$ ) is $W_{k}$-equivalent to $s_{k}$.

Due to the construction of retesting sequences, we also check that (X-1) holds for each transition from each state $s_{j} \notin S_{u}$. Thus, if $M_{I}{ }^{\prime}$ passes the test, then the mapping $h$ satisfies (X-1), i.e. $M_{I}{ }^{\prime}$ is quasi-equivalent to $M S^{\prime} \square$ 
As an application example for Case-3 with the HIS method, we add to the given specification a new state $s^{\prime} 4$ and its corresponding incoming and outgoing edges producing the modified specification $M S^{\prime}$ shown in Fig. 2.

The state cover set of $M_{S}^{\prime}$ is $Q^{\prime}=\{\varepsilon, b, c, b c\}$. We consider each incoming and outgoing transition of the added state (here $s^{\prime} 4$ ) as a modified transition. Therefore, the modified transitions of $M S^{\prime}$ are $\left(s^{\prime} 2-c / f->s^{\prime} 4\right),\left(s^{\prime} 4-b / f->s^{\prime} 4\right)$, $\left(s^{\prime} 4-a l e->s^{\prime} 4\right)$, and ( $\left.s^{\prime} 4-c / f->s^{\prime} 3\right)$.

According to Case-3, we add to the set $S_{u}$ states $s_{1}^{\prime}, s_{2}^{\prime}$, and $s_{3}^{\prime}$ since these states are reachable through unmodified transitions and there exists a state identifier for each of these states that does not pass through modified transitions. In this example, the sequence $b b$ is such an identifier. In fact, we have the following input/output sequences in response to $b b$. For state $s^{\prime} 1, f f$. For state $s_{2}^{\prime}, f e$. For state $s_{3}^{\prime}, e f$, and for state $s_{4}^{\prime}, e e$.

These states, i.e., $s^{\prime} 1, s^{\prime} 2$, and $s^{\prime} 3$, do not need to be re-identified in the new implementation. In order to re-identify the added state $s^{\prime} 4$, the re-test sequence $r . \alpha_{4} . W_{4}=r . b c . b b$ is selected using Formula (3-1a). If the modified implementation passes this sequence then there is a one-to-one mapping between states of the modified specification and implementation that are $b b$ equivalent.

Afterwards, in order to test the modified transition $\left(s^{\prime} 2-c / f->s^{\prime} 4\right)$ whose head state $s_{2}^{\prime}$ is in $S_{u}$, the sequence r. $\alpha_{2} . c . W_{4}=r . b . c . b b$ is selected using Formula (1-a). Moreover, the following re-testing test sequences are selected using Formula (3-1c) in order to check the outgoing transitions from state $s_{4}^{\prime} \in S S_{u}: r . \alpha_{4} \cdot a \cdot W_{4}+r . \alpha_{4} \cdot b \cdot W_{4}+r . \alpha_{4} . c . W_{3}=r . b c \cdot a \cdot b b+r . b c . b . b b+r . b c . c . b b$

Consequently, the re-testing test suite has sequences of total length 18. The traditional HIS method derives a test suite of length 32 if the whole specification of $M S^{\prime}$ is considered for test derivation.

\section{FURTHER RESEARCH WORK}

We have extended the re-testing methods presented in this paper for the case when the system implementation may have more states than its specification. Moreover, we are adapting these methods for a system modeled as an Extended Finite State Machine (EFSM). The problem here is to find an appropriate way for re-testing both the control flow and the data flow parts of a modified EFSM. Finally, we are investigating how the retesting methods can be applied to a labeled transition system (LTS). 


\section{REFERENCES}

[1] T. S. Chow, 'Test design modeled by finite-state machines'. IEEE Trans. SE-4, No.3, pp. 178-187, 1978.

[2] K. El-Fakih, N, Yevtushenko, and G. v. Bochmann, 'Re-testing Based on Finite State Model', Technical Report, University of Ottawa, 2001.

[3] S. Fujiwara, G. v. Bochmann, F. Khendek, M. Amalou, and A. Ghedamsi, 'Test selection based on finite state models', IEEE Trans. SE-17, No. 6, 591-603, 1991.

[4] A. Gill, Introduction to the Theory of Finite-State Machines. McGraw-Hill, 1962.

[5] I.Koufareva, A.Petrenko, N.Yevtushenko. Test generation driven by user-defined fault models. In Proc. of the IFIP TC6 12th International Workshop on Testing of Communicating Systems, Hungary, pp. 215-233, 1999.

[6] A. Petrenko, Checking experiments with protocol machines, Proceedings of the IFIP Fourth International Workshop on Protocol Test Systems, the Netherlands, 1991, pp. 83-94.

[7] A. Petrenko and N. Yevtushenko, 'Test suite generation for a FSM with a given type of implementation errors', Proc. of the $12^{\text {th }}$ IWPSTV, pp. 229-243, 1992.

[8] A. Petrenko, N. Yevtushenko, A. Lebedev, and A. Das, 'Nondeterministic state machines in protocol conformance testing', In Proc. of the IFIP Sixth International Workshop on Protocol Test Systems, France, pp. 363-378, 1993.

[9] K. Sabnani and A. Dahbura,'A protocol test generation procedure'. Computer Networks and ISDN Systems, Vol. 15, No. 4, 285-297, 1988.

[10] S. Schach, Software Engineering, Boston:Aksen Assoc., 1992.

[11] M. Yannakakis and D. Lee, 'Testing finite state machines: fault detection', Journal of Computer and System Sciences, 50, 1995, pp. 209-227, 1995. 